\title{
Valproate semisodium-induced encephalopathy: diet and polypharmacy interactions
}

\author{
Nuno Rodrigues Silva, ${ }^{1}$ Ângela Venâncio ${ }^{1}$
}

The Psychiatrist (2012), 36, 137-139, doi: 10.1192/pb.bp.111.035261

${ }^{1}$ Gaia/Espinho Hospital Center, Vila Nova de Gaia, Portugal

Correspondence to Nuno Rodrigues Silva (nuno.rodrigues.silva@chvng. min-saude.pt)

First received 10 May 2011, final revision 16 Sep 2011, accepted 5 Oct 2011
Summary Despite extensive use of valproate in neurology and psychiatry, valproateinduced encephalopathy is a rarely reported complication. Although initially reported in the paediatric population, case reports are growing in the adult population. Clinicians need to be aware of this potentially life-threatening complication. We report a case in a 37-year-old woman with bipolar I disorder with previously uncomplicated valproate use, who developed encephalopathy when valproate was restarted a few years later. The patient has provided consent for publication.

Declaration of interest None.

\section{Case report}

Mrs M was a 37-year-old woman diagnosed with bipolar I disorder when she was 22 years old. Mrs M was taken to a psychiatric emergency unit 3 days after progressive worsening, with insomnia, agitation, aggressiveness, euphoria and at times anger, disinhibition, disorganised speech, pressure of speech, and mystical, grandiose and persecutory related delusions. She had no insight regarding her clinical situation and refused to take any medication, and was therefore involuntarily admitted to our psychiatric unit.

Mrs M had been admitted to hospital previously as a result of maniac episodes, once in 2003 and twice in 2006, both related to non-adherence to treatment. She had been taking medication for a number of years. Originally, she was prescribed valproate semisodium $(1000 \mathrm{mg} / \mathrm{day}$ for 10 months) and olanzapine, and no side-effects were reported. Following the last time she was admitted to hospital in 2006 she had been stabilised on lamotrigine plus quetiapine, although side-effects had prompted dose adjustment. Eight months before the current admission to hospital the patient quitted treatment and consultations and started a homeopathic treatment as she wanted to become pregnant. There was no history of substances misuse. In her medical history the only other factor of note was the presence of thyroid nodules with normal thyroid function and she was not on medication. A year before her transfer to our unit she had started a vegetarian diet with infrequent intake of fish (once a week) and meat (once or twice a month).

On transfer to our unit (day 0), her initial therapeutic regimen included risperidone $3 \mathrm{mg}$ (once a day), valproate semisodium $250 \mathrm{mg}$ (twice a day) and lorazepam $1 \mathrm{mg}$ (once a day) plus $2.5 \mathrm{mg}$ before bedtime. Valproate was increased to $500 \mathrm{mg}$ (twice a day) 3 days later. In the following days Mrs M's mood improved, although some lability was still noted. An urge to move became apparent and the insomnia persisted. Biperiden $4 \mathrm{mg}$ (twice a day) and trazodone
$100 \mathrm{mg}$ (once a day) were started to address these issues. The akathisia and insomnia soon improved.

On day 9 she presented lethargic, poorly responsive to external stimuli and her pupils were wide. Vital signs were normal and there was no muscle rigidity, tongue bites or faecal/urine incontinence. She was transferred to the emergency unit. The laboratory results were unremarkable, with a normal complete blood count, glycaemia, renal function, electrolytes, liver enzymes and albumin, except for an elevated C-reactive protein level $(5.02 \mathrm{mg} / \mathrm{l}$ ) (normal range: $0.01-0.5 \mathrm{mg} / \mathrm{l}$ ). Arterial blood gases were also within the normal range. Valproate levels were within the therapeutic range $(98.4 \mu \mathrm{g} / \mathrm{ml})$, although close to the upper limit (normal range: 50-100 $\mu \mathrm{g} / \mathrm{ml}$ ). Computed tomography of the brain was normal. Within a few hours Mrs M returned to our unit. Valproate was reduced to $750 \mathrm{mg} /$ day (250 $\mathrm{mg}$ in the morning $+500 \mathrm{mg}$ at night) and biperiden to $2 \mathrm{mg}$ (twice a day).

In the following days, Mrs M progressively improved but there was still some fluctuating confusion, disorganised thought and facial misidentifications. Valproate was further reduced to $250 \mathrm{mg}$ (twice a day) on day 14 . She was seen by a neurologist who did not find signs of a specific aetiology.

On day 16, Mrs M experienced visual hallucinations and the next day she became obtunded. Blood tests were performed (including calcium, creatine kinase and ammonia) and were unremarkable except for the ammonia level, which was $70.8 \mu \mathrm{g} / \mathrm{dl}$ (normal range: $15-45 \mu \mathrm{g} / \mathrm{dl}$ ). Valproate was immediately stopped and lactulose initiated (30 $\mathrm{ml}$ once daily; $10 \mathrm{~g} / 15 \mathrm{ml}$ twice a day). In addition, risperidone was switched to quetiapine $400 \mathrm{mg}$. Gradual improvement was evident over the next days. Ammonia levels were measured every 2 days and normalised 4 days after discontinuation of valproate ( $17.5 \mu \mathrm{g} / \mathrm{dl})$. Unfortunately, an electroencephalogram was only possible on day 24 and showed no abnormalities. Magnetic resonance imaging (MRI) of the brain was also performed and showed small $T_{2}$-hyperintense areas bilaterally over frontoparietal white 
matter reflecting areas of gliosis, with no involvement in the basal ganglia or the cerebellum.

On day 32 , Mrs M was clinically stabilised and was discharged home. Of note, Mrs M experienced temporary amnesia while in the encephalopathic state and has no recollection of what happened then.

\section{Discussion}

This case report describes an adult woman who, despite uncomplicated valproate use 7 years previously and present total serum valproate levels within the normal range, developed valproate-induced hyperammonaemic encephalopathy. This occurred less than 9 days after the drug was restarted. When first suspected the dose of valproate was reduced, but after a slight improvement the woman's condition deteriorated even further after 7 days and at this time valproate was discontinued. The distinction between psychotic symptoms of disorganisation and delirium is often problematic, delaying the diagnosis, as was the case with our patient.

Valproate-induced encephalopathy can present with the following signs and symptoms: ${ }^{1}$

- acute-onset impaired consciousness level, disorientation, shifting attention, memory impairment;

- visual hallucinations;

- tremor, ataxia, perseveration, vomiting;

- seizures, coma, death;

- raised ammonia level, normal routine tests (complete blood count, renal function, electrolytes, inflammatory markers).

In our patient the acute confusional state after valproate initiation, its resolution after discontinuation, an associated elevated ammonia level and the MRI findings strongly suggest a causal relationship between valproate and the encephalopatic state. But why did this happen at this time? Were new factors present that could explain this complication?

The hypothesised underlying mechanism of valproateinduced hyperammonaemic encephalopathy, although not completely understood in animal studies, indicates both hepatic and renal involvement, with the former appearing to have the major impact. ${ }^{1,2}$ Within the kidney, valproate and its metabolites increase ammonia production by stimulating the uptake of glutamine and glutamate, thereby resulting in an increase in the renal venous release of ammonia. ${ }^{1}$ In hepatic mitochondria, valproate binds to free carnitine and co-enzyme A, decreasing their availability and ultimately affecting the urea cycle and increasing ammonia levels., ${ }^{1,3}$ Within the brain, hyperammonaemia leads to higher production of glutamine in astrocytes, which promotes oedema and subsequent dysfunction. ${ }^{4}$

In previously reported cases, hyperammonaemia mediated by valproic acid occured at both therapeutic and supratherapeutic levels. ${ }^{5}$ This could be the result of increased free valproic acid levels in the presence of normal total valproic acid levels, which is routinely measured. Free and total valproic acid levels share a linear relationship when the total valproic acid level is in the low therapeutic range, but at higher total valproic acid levels there is an exponential rise. ${ }^{6}$ This could be related to valproic acid binding site saturation in albumin, with a further increase in total valproic acid levels causing a disporportionate rise in free valproic acid. In this regard, monitoring free valproic acid levels may be more useful, as suggested by Modjtahedi. ${ }^{6}$

Possible risk factors for valproate-induced encephalopathy include dietary restrictions, polypharmacy and carnitine or urea cycle genetic disorders. Our patient had a very low intake of meat and dairy products. In two previously reported cases, the individuals were vegetarians. ${ }^{7}$ Animal protein is the source of carnitine for most people. In general, $20-200 \mathrm{mg} /$ day are ingested in people with an omnivorous diet, whereas strict vegetarians may ingest $1 \mathrm{mg} /$ day. $^{8}$ Carnitine is also synthesised within the body in small quantities. ${ }^{7}$

It has been previously reported that both strict vegetarians and ovo-lacto-vegetarians have significantly lower plasma carnitine concentrations and markedly lower urinary carnitine excretion compared with a group with a mixed diet. ${ }^{9}$ However, the authors found that plasma carnitine levels, although lower than that in the controls, were within the normal range (of interest, children exhibited greater differences, possibly explaining the more abundant literature reporting valproate-induced encephalopathy in this population). Humans can synthesise carnitine in small amounts, which combined with efficient reabsorption by the kidneys (95\%), is sufficient by itself to prevent carnitine deficiency in otherwise healthy people. ${ }^{8}$ However, carnitine equilibrium may be overwhelmed in susceptible people (e.g. vegetarians, who depend mostly on carnitine biosynthesis) due to valproate binding to free carnitine, ultimately leading to an increase in ammonia levels in the blood.

Polypharmacy can also be a significant issue whenever individuals are on medications that influence valproate metabolism or transport. Valproate is highly bound to albumin and is concentration dependent and inversely correlated to free valproate. $^{10}$ In a report of two cases, risperidone was related to increased valproic acid levels in the blood, possibly through competition for protein binding sites. ${ }^{11}$ It was also speculated that lorazepam could interact with valproate. Although there is evidence that valproate affects lorazepam metabolism, whether the opposite is the case is not so clear and this combination has long been used with safety. ${ }^{12}$

Clinicians should be alert to the following factors when considering a diagnosis of valproate-induced encephalopathy.

- Normotherapeutic valproate serum levels do not discount valproate-induced encephalopathy.

- Uncomplicated valproate use in the past does not exclude future complications.

- Accurate distinction between delirium and psychosis with disorganised symptoms is paramount and should provide clinicians with an early suspicion of encephalopathy.

- Valproate initiation and maintenance, and the concomitant use of other drugs should be scrutinised.

With increasing evidence relating to valproate and encephalopathy in psychiatric settings, it should be current 
Box 1 Possible risk factors for valproate-induced encephalopathy

- Diet:

- vegetarianism ${ }^{5,11}$

- malnutrition, low caloric intake ${ }^{13}$

- high nitrogen load ${ }^{13,14}$

- parenteral nutrition ${ }^{15}$

- Urea cycle disorders ${ }^{16}$

- Immature hepatic function (infancy) ${ }^{17}$

- Hypoalbuminaemia

- Drug interactions:

- risperidone ${ }^{11}$

- phenobarbital $^{18,19}$

- phenytoin $^{19}$

- topiramate 20

- aspirin $^{21}$

- Age

- Complicating medical conditions:

- renal impairment

- neurological disorders ${ }^{15}$

practice to assess and monitor individuals at higher risk (Box 1) for this still uncommon but serious complication.

\section{About the authors}

Nuno Rodrigues Silva is a psychiatry resident, in the Department of Psychiatry and Mental Health at Gaia/Espinho Hospital Center, Vila Nova de Gaia, Portugal. Ângela Venâncio is a consultant psychiatrist, in the Department of Psychiatry and Mental Health at Gaia/Espinho Hospital Center, Vila Nova de Gaia, Portugal.

\section{References}

1 Verrotti A, Trotta D, Morgese G, Chiarelli F. Valproate-induced hyperammonemic encephalopathy. Metab Brain Dis 2002; 17: 267-73.

2 Elharmi M, Ferrier B, Martin M, Baverel G. Effect of valproate, sodium 2propyl-4-pentenoate and sodium 2-propyl-2-pentoate on renal substrate uptake and ammoniagenesis in the rat. J Pharmacol Exp Ther 1993; 266: 89-96.
3 McCall M, Bourgeois J. Valproic acid-induced hyperammonemia: a case report. J Clin Psychopharmacol 2004; 24: 521-6.

4 Brusilow S. Hyperammonemic encephalopathy. Medicine (Baltimore) 2002; 81: 240-9.

5 Carr RB, Shrewsbury K. Hyperammonemia due to valproic acid in the psychiatric setting. Am J Psychiatry 2007; 164: 1020-7.

6 Modjtahedi B. Hyperammonemia and valproic acid-induced encephalopathy. Am J Psychiatry 2007; 164: 1912-3.

7 Stanley CA. Carnitine deficiency disorders in children. Ann N Y Acad Sci 2004; 1033: 42-51.

8 Rebouche C. Modern Nutrition in Health and Disease (9th edn). Lippincott, Williams \& Wilkins, 1999.

9 Lombard K, Olson A, Nelson S, Rebouche C. Carnitine status of lactoovovegetarians and strict vegetarians adults and children. Am J Clin Nutr 1989; 50: 301-6.

10 Anderson G, Gidal B, Hendryx R, Awan A, Temkin N, Wilensky A, et al. Decreased plasma protein binding of valproate in patients with acute head trauma. Br J Clin Pharmacol 1994; 37: 559-62.

11 Carlson T, Reynolds C, Caplan R. Case report: valproic acid and risperidone treatment leading to development of hyperammonemia and mania. J Am Acad Child Adolesc Psychiatry 2007; 46: 356-61.

12 Kimmel R, Irwin S, Meyer J. Valproic acid-associated hyperammonemic encephalopathy: a case report from the psychiatric setting. Int Clin Psychopharmacol 2005; 20: 57-8.

13 Laub MC. Nutritional influence on serum ammonia in young patients receiving sodium valproate. Epilepsia 1986; 27: 55-9.

14 Gidal BE, Inglese CM, Meyer JF, Pitterle ME, Antonopolous J, Rust RS Diet- and valproate-induced transient hyperammonemia: effect of L-carnitine. Pediatr Neurol 1997; 16: 301-5.

15 Coulter DL. Carnitine deficiency in epilepsy: risk factors and treatment. J Child Neurol 1995; 10 (suppl): s32-3.

16 Oechsner M, Steen C, Sturenburg HJ, Kohlschutter A. Hyperammonaemic encephalopathy after initiation of valproate therapy in unrecognised ornithine transcarbamylase deficiency. J Neurol Neurosurg Psychiatry 1998; 64: 680-2.

17 Raskind JY, El-Chaar GM. The role of carnitine supplementation during valproic acid therapy. Ann Pharmacother 2000; 34: 630-8.

18 Zaccara G, Paganini M, Campostrini R, Moroni F, Valenza T, Messori A et al. Effect of associated antiepileptic treatment on valproate-induced hyperammonemia. Ther Drug Monit 1985; 7: 185-90.

19 Warter JM, Marescaux C, Brandt C, Rumbach L, Micheletti G Chabrier G. Sodium valproate associated with phenobarbital: effects on ammonia metabolism in humans. Epilepsia 1983; 24: 628-33.

20 Hamer HM, Knake S, Schomburg U, Rosenow F. Valproate-induced hyperammonemic encephalopathy in the presence of topiramate. Neurology 2000; 54: 230-2.

21 Sandson NB, Marcucci C, Bourke DL, Smith-Lamacchia R. An interaction between aspirin and valproate: the relevance of plasma protein displacement drug-drug interactions. Am J Psychiatry 2006; 163 : 1891-6. 